\title{
A Paraconsistentist Approach to Chisholm’s Paradox
}

\author{
Marcelo Esteban Coniglio \\ Newton Marques Peron \\ State University of Campinas
}

\begin{abstract}
The Logics of Deontic (In)Consistency (LDI's) can be considered as the deontic counterpart of the paraconsistent logics known as Logics of Formal (In)Consistency. This paper introduces and studies new LDI's and other paraconsistent deontic logics with different properties: systems tolerant to contradictory obligations; systems in which contradictory obligations trivialize; and a bimodal paraconsistent deontic logic combining the features of previous systems. These logics are used to analyze the well-known Chisholm's paradox, taking profit of the fact that, besides contradictory obligations do not trivialize in LDI's, several logical dependencies of classical logic are blocked in the context of LDI's, allowing to dissolve the paradox.
\end{abstract}

Keywords: Paraconsistent logic, deontic logic, deontic paradoxes, chisholm's paradox, moral dilemmas, logics of formal inconsistency.

\section{Introduction}

The phenomenon of inconsistency has catastrophic consequences in theories based on classical logic. There is a simple reason to this: in the context of classical logic, a contradiction like $\alpha$ and $\neg \alpha$ trivializes the whole theory, sinc everything is logically derived from a contradiction. Paraconsistent logics, developed independently by da Costa 1963 and Jaśkowski 1948, are logics tolerant to contradictions, and so they admit non-trivial inconsistent (closed) theories.

The Logics of Formal (In)Consistency (LFI's), introduced in Carnielli \& Marcos 2002 and also studied in Carnielli, Coniglio \& Marcos 2007, are a special class of paraconsistent logics in which there is a formal distinction between notions such as contradiction, inconsistency and triviality. This is attained by the use in the formal language of connectives for consistency and inconsistency.

A phenomenon analogous to inconsistency takes place with deontic logics, in the context of moral dilemmas (see, for instance, McConnell 2006). If O denotes the deontic "obligation" operator, a moral dilemma is a situation of conflicting or contradictory obligations of the form $\mathrm{O} \alpha$ and $\mathrm{O} \neg \alpha$. Note that this is not a particular case of contradictoriness like $\mathrm{O} \alpha$ and $\neg \mathrm{O} \alpha$, but a different (however related) situation. In the presence of conflicting obligations, classical deontic logics trivialize,

Principia 13(3): 299-326 (2009).

Published by NEL — Epistemology and Logic Research Group, Federal University of Santa Catarina (UFSC), Brazil. 
in the same manner as classical logic trivializes when a contradiction occurs. This similarity justify a paraconsistentist approach to deontic paradoxes.

The first paraconsistent deontic system, called $C_{1}^{D}$, was introduced in da Costa \& Carnielli 1986 with the aim of analyzing moral dilemmas. It is a deontic extension of da Costa's logic $C_{1}$, being also suitable to the study of deontic paradoxes in general.

The Logics of Deontic (In)Consistency (LDI's) were firstly introduced in Coniglio 2007, by considering two deontic systems, DmbC and DLFI1, based on two different LFI's. It was shown that these systems are appropriate to analyze the well-known deontic paradox of Chisholm introduced in Chisholm 1963.

In Peron \& Coniglio 2008 two new LDI's were introduced, SDmbC and BDmbC, enlarging the possibilities of analysis of Chisholm's paradox, and in Peron 2009 it was presented the distinction between LDI's and deontic LFI's, among other results.

The present paper extends our previous discussions on the subject, in particular the analysis of Chisholm's paradox in this context. Moreover, two new paraconsistent deontic systems are introduced, which can be considered, in some sense, as minimal: DPI, a LDI which is not a LFI, and SDPI, both a LDI and a LFI such that conflicting obligations trivialize, provided that the involved sentence is deontically consistent. Concerning Chisholm's paradox, it is concluded here that the violation of the Principle of Deontic Consistency is not the unique source of problems in deontic paradoxes based on contrary-to-duty obligations. Indeed, the rigidity of classical logic, in which too much sentences are logically identified, originates unnecessary logical dependencies among the premises of a given information set, and then several plausible formalizations of the given premises must be discarded. The use of a richer language based on a more flexible logic allows more translations from the natural language to the formal one, and so the paradox can vanish. If, in addition, the Principle of Deontic Consistency is weakened, there are still more possibilities to avoid the paradox.

The organization of the paper is as follows: in Section 1 the basic notions concerning paraconsistency in general and LFI's in particular are briefly recalled. In Section 2 it is firstly analyzed the Standard Deontic Logic SDL and then the LDI's are introduced, describing succinctly the main systems to be studied in the paper. Section 3 is devoted to the analysis of Chisholm's paradox under the perspective of some systems, namely SDL, DmbC, SDmbC, DPI, SDPI and BDmbC. Finally, Section 4 concludes with a brief evaluation of what was done in this paper.

\section{Paraconsistency and Formal (In)Consistency}

Da Costa's approach to paraconsistency is based on the idea that certain sentences of the object language can be marked as being "classical" or "well-behaved" (cf. da

Principia 13(3): 299-326 (2009). 
Costa 1963). More precisely, in a logical context in which contradictions are possible, in the sense that a contradiction does not necessarily trivialize, it is possible to define or to assume as hypothesis inside a formal deduction that an specific sentence, say $\alpha$, has classical properties. The classical (or well) behavior of $\alpha$ is another sentence, $\neg(\alpha \wedge \neg \alpha)$, usually denoted by $\alpha^{\circ}$. In order to simplify the exposition we limit ourselves to the logic $C_{1}$, the first logic in the decreasing hierarchy of paraconsistent $\operatorname{logics}\left(C_{n}\right)_{n \geq 1}$. Being so, in general

$$
\alpha, \neg \alpha \nvdash \beta
$$

for some $\beta$, but always

$$
\alpha^{\circ}, \alpha, \neg \alpha \vdash \beta
$$

for every $\beta$ (here, ' $\vdash$ ' is the symbol for the consequence relation, and so ' $\forall$ ' means "does not follow"). That is, contradictions do not necessarily trivialize, but contradictions of classically (or well) behaved sentences always trivialize (or "explode").

There is another way of interpreting da Costa's approach to paraconsistency: a contradiction is not enough to trivialize, but a contradiction of a contradiction trivializes:

$$
\alpha, \neg \alpha \nvdash \beta
$$

for some $\beta$, but always

$$
(\alpha \wedge \neg \alpha), \neg(\alpha \wedge \neg \alpha) \vdash \beta
$$

for every $\beta$. In other words, contradictions are not logically trivial, but a contradiction involving a contradiction is!

It can be observed that the statement that $\alpha$ is well-behaved, namely $\alpha^{\circ}$, is expressed in terms of the other connectives of the object language. Thus, if $(\alpha \wedge \neg \alpha)$ can be read as " $\alpha$ is contradictory" then $\alpha^{\circ}$ says that " $\alpha$ is not contradictory". Being so, non-contradictoriness is equivalent to well-behaviorness, or classicality, or consistency. But, are the notions of non-contradiction and consistency (or, dually, the notions of contradiction and inconsistency) necessarily the same?

This is the standpoint of Logics of Formal (In)Consistency (in short, LFI's), introduced in Carnielli \& Marcos 2002 with the aim of generalizing da Costa's C-systems. In this approach the notion of well-behavior or consistency of a sentence $\alpha$ is represented by the sentence $\circ \alpha$, where $\circ$ is a primitive connective of the object language (and, different to the logics $C_{n}$, in general this connective cannot be expressed in terms of the others). Dually, it is possible to consider a inconsistency connective • as a primitive, such that the interdefinability laws $\circ \alpha \equiv \neg \bullet \alpha$ and $\bullet \alpha \equiv \neg \circ \alpha$ hold in general.

In term of consistency, LFI's has the following property (compare with $C_{1}$ above):

$$
\alpha, \neg \alpha \forall \beta
$$

Principia 13(3): 299-326 (2009). 
for some $\beta$, but always

$$
\circ \alpha, \alpha, \neg \alpha \vdash \beta
$$

for every $\beta$. But, different to da Costa's systems, the consistency operator $\circ$ is primitive and is not necessarily identical to non-contradiction: in the weaker LFI's, $\circ \alpha$ and $\neg(\alpha \wedge \neg \alpha)$ are not equivalent. Moreover, in general

$$
\begin{aligned}
& \circ \alpha \vdash \neg(\alpha \wedge \neg \alpha) \text { but } \neg(\alpha \wedge \neg \alpha) \forall \circ \alpha ; \\
& \neg \circ \alpha \vdash(\alpha \wedge \neg \alpha) \text { but }(\alpha \wedge \neg \alpha) \forall \neg \circ \alpha .
\end{aligned}
$$

Thus, in the context of LFI's the notions of consistency and non-contradiction, as well as the notions of inconsistency and contradiction, can be separated. The steps through the definition of LFI's are briefly outlined below (the interested reader can consult Carnielli \& Marcos 2002 and Carnielli, Coniglio \& Marcos 2007).

Let $\mathbf{L}$ be a (propositional) logic having a negation connective $\neg$. A theory $\Gamma$ of $\mathbf{L}$ (that is, a set $\Gamma$ of sentences of $\mathbf{L}$ ) is contradictory if $\Gamma \vdash \alpha$ and $\Gamma \vdash \neg \alpha$ for some sentence $\alpha$. Obviously, $\Gamma$ is not contradictory if and only if, for every $\alpha$, either $\Gamma \nvdash \alpha$ or $\Gamma \nvdash \neg \alpha$. A theory $\Gamma$ is trivial if $\Gamma \vdash \alpha$ for every $\alpha$, and is non-trivial otherwise. If $\Gamma, \alpha, \neg \alpha \vdash \beta$ for every $\alpha$ and $\beta$ the theory $\Gamma$ is said to be explosive. A logic $\mathrm{L}$ is non-trivial if it has a non-trivial theory, and it is explosive if every theory is explosive (equivalently, if $\alpha, \neg \alpha \vdash \beta$ for every $\alpha$ and $\beta$ ). A logic $\mathbf{L}$ is paraconsistent if it is non-trivial and non-explosive.

Let $\bar{\bigcirc}(p)$ be a set of sentences depending exactly on the propositional letter $p$ such that

(a) $\bar{\bigcirc}(\alpha), \alpha \nvdash \beta$

(b) $\bar{\bigcirc}\left(\alpha^{\prime}\right), \neg \alpha^{\prime} \nvdash \beta^{\prime}$

for some $\alpha, \alpha^{\prime}, \beta$ and $\beta^{\prime}$. A logic L is gently explosive (w.r.t. $\bar{\bigcirc}(p)$ ) if

(c) $\bar{\bigcirc}(\alpha), \alpha, \neg \alpha \vdash \beta$

for every $\alpha$ and $\beta$. Given a sentence $\alpha$, the set $\bar{\bigcirc}(\alpha)$ expresses the consistence of $\alpha$ relative to logic $\mathbf{L}$ (and negation $\neg$ ). Whenever the set $\bar{\bigcirc}(p)$ is a singleton, the unique element of it will be denoted by op and then the sentence $\circ \alpha$ denotes the consistency of $\alpha$, where $\circ$ is called a consistency operator. By the very definition of consistency operator, $\circ \alpha, \alpha, \neg \alpha \vdash \beta$ for every $\alpha$ and $\beta$.

Definition 1.1. A Logic of Formal Inconsistency (LFI) is a paraconsistent logic having a set $\bar{\bigcirc}(p)$ satisfying properties (a), (b) and (c) as above.

Principia 13(3): 299-326 (2009). 
It is immediate to see that logic $C_{1}$ is a LFI with $\circ \alpha=\alpha^{\circ}=\neg(\alpha \wedge \neg \alpha)$. Moreover, every $\operatorname{logic} C_{n}$ (for $n \geq 1$ ) is a LFI with an appropriate definition of the consistency operator $\circ_{n}$. In fact, consider the following recursive definition: $\alpha^{1}=\alpha^{\circ}$, and $\alpha^{n+1}=$ $\left(\alpha^{n}\right)^{\circ}$ (for $n \geq 1$ ). Finally, let $\alpha^{(1)}=\alpha^{\circ}$ and $\alpha^{(n+1)}=\alpha^{(n)} \wedge \alpha^{n+1}$ (for $n \geq 1$ ). Then, consistency in logic $C_{n}$ is given by the operator $\circ_{n} p=p^{(n)}$ or, equivalently, by the set $\bar{\bigcirc}_{n}(p)=\left\{p^{1}, p^{2}, \ldots, p^{n}\right\}$ (for $n \geq 1$ ). It is interesting to observe that the logic $C_{\text {Lim }}$ (cf. Carnielli \& Marcos 1999), the deductive limit of the hierarchy $\left(C_{n}\right)_{n \geq 1}$, is a LFI in which consistency is expressed by the infinite set $\bar{\bigcirc}(p)=\left\{p^{1}, p^{2}, p^{3}, \ldots\right\}$. That is, there are LFI's in which consistency is expressed by an infinite set of sentences instead of a single consistency operator.

The simplest LFI introduced in Carnielli \& Marcos 2002 is called mbC, defined as follows:

Definition 1.2. The logic mbC is defined over the language $\circ, \neg, \wedge, \vee, \rightarrow$ by means of the following:

\section{Axiom schemas:}

(Ax1) $\alpha \rightarrow(\beta \rightarrow \alpha)$

(Ax2) $(\alpha \rightarrow \beta) \rightarrow(\alpha \rightarrow(\beta \rightarrow \gamma)) \rightarrow(\alpha \rightarrow \gamma))$

(Ax3) $\alpha \rightarrow(\beta \rightarrow(\alpha \wedge \beta))$

(Ax4) $(\alpha \wedge \beta) \rightarrow \alpha$

(Ax5) $(\alpha \wedge \beta) \rightarrow \beta$

(Ax6) $\alpha \rightarrow(\alpha \vee \beta)$

(Ax7) $\beta \rightarrow(\alpha \vee \beta)$

(Ax8) $(\alpha \rightarrow \gamma) \rightarrow((\beta \rightarrow \gamma) \rightarrow((\alpha \vee \beta) \rightarrow \gamma))$

(Ax9) $\alpha \vee(\alpha \rightarrow \beta)$

(Ax10) $\alpha \vee \neg \alpha$

(bc1) $\circ \alpha \rightarrow(\alpha \rightarrow(\neg \alpha \rightarrow \beta))$

Inference rule:

(MP) $\frac{\alpha \quad \alpha \rightarrow \beta}{\beta}$ 
From now on, the set of sentences defined over the basic language $\neg, \wedge, \vee, \rightarrow$ will be denoted by For, and the set of sentences of mbC (which is obtained by adding the unary connective o) will be denoted by For ${ }^{\circ}$.

It is worth noting that the system defined over For by means of (Ax1)-(Ax9) and (MP) is the so-called Positive Classical Logic, denoted by PCL. Classical Logic CL defined over For is obtained from PCL by adding (Ax10) and the "explosion law" (exp) $\alpha \rightarrow(\neg \alpha \rightarrow \beta)$

Of course a version of CL over $F_{0}^{\circ}{ }^{\circ}$, called eCL, can be obtained from mbC by adding the axiom schema $\circ \alpha$; in this sense CL is a deductive extension of $\mathbf{m b C}$ in which every sentence is consistent (and so (bc1) collapses to (exp)). On the other hand, $\perp=_{\text {def }} \circ \alpha \wedge(\alpha \wedge \neg \alpha)$ is a bottom sentence in $\mathbf{m b C}$, that is, $\perp \vdash_{\mathrm{mbC}} \beta$ for every $\beta$ and so $\sim \alpha=_{\text {def }}(\alpha \rightarrow \perp)$ is a classical negation in $\mathbf{m b C}$. Therefore mbC defined over the set of sentences For ${ }^{\circ \sim}$ generated by $\sim, \circ, \neg, \wedge, \vee, \rightarrow$ (that is, with classical negation $\sim$ as a primitive) can be seen as the conservative extension of CL (now defined over the language $\sim, \wedge, \vee, \rightarrow$ ) obtained by adding axiom schemas (Ax10), (bc1) and $\sim \alpha \leftrightarrow(\alpha \rightarrow \perp)$ where, as usual, $(\alpha \leftrightarrow \beta)$ stands for $(\alpha \rightarrow \beta) \wedge(\beta \rightarrow \alpha)$.

The logic mbC (as most of the LFI's presented in Carnielli \& Marcos 2002 and Carnielli, Coniglio \& Marcos 2007) does not satisfy the replacement property, namely:

$$
\text { if } \alpha \dashv \beta \text { then } \varphi(\alpha) \dashv \varphi(\beta)
$$

for every $\alpha, \beta, \varphi$ (here, $\alpha \dashv \beta$ means that $\alpha$ and $\beta$ are interderivable, that is, $\alpha \vdash \beta$ and $\beta \vdash \alpha)$. For instance, $(p \wedge q)$ is interderivable with $(q \wedge p)$, but $\neg(p \wedge$ $q$ ) is not interderivable with $\neg(q \wedge p)$; analogously, $\circ(p \wedge q)$ is not interderivable with $\circ(q \wedge p)$. This means that $\neg$ and $\circ$ are non-truth-functional connectives and so semantics is not a trivial issue for mbC (nor for the other LFI's with non-truthfunctional connectives). In Carnielli, Coniglio \& Marcos 2007 the following twovalued semantics was proposed for $\mathrm{mbC}$ :

Definition 1.3. Let $\mathbf{2}=\{0,1\}$ be a set of truth-values, where 1 denotes "true" and 0 denotes "false". A $m b C$-valuation is a mapping $v:$ For $^{\circ} \longrightarrow \mathbf{2}$ satisfying the following clauses:

(v1) $v(\alpha \wedge \beta)=1$ iff $v(\alpha)=v(\beta)=1$,

(v2) $v(\alpha \vee \beta)=0$ iff $v(\alpha)=v(\beta)=0$,

(v3) $v(\alpha \rightarrow \beta)=0$ iff $v(\alpha)=1$ and $v(\beta)=0$,

(v4) $v(\neg \alpha)=1$ implies $v(\alpha)=0$,

(v5) $v(\circ \alpha)=1$ implies $v(\alpha)=0$ or $v(\neg \alpha)=0$. 
As usual, we define $\Gamma \vDash_{\text {mbC }} \alpha$ iff $v(\alpha)=1$ for every mbC-valuation $v$ such that $v(\Gamma) \subseteq\{1\}$. Thus, the logic $\mathbf{m b C}$ is sound and complete for its valuation semantics, cf. Carnielli, Coniglio \& Marcos 2007:

Theorem 1.4. Let $\Gamma \cup\{\alpha\}$ be a set of sentences in For ${ }^{\circ}$. Then, $\Gamma \vdash_{\mathbf{m b C}} \alpha$ if and only if $\Gamma \vDash_{\text {mbC }} \alpha$.

\section{Logics of Deontic (In)Consistency}

\subsection{Standard Deontic Logic and Paraconsistency}

Deontic logics study notions such as "obligation", "permission", "prohibition" and similar concepts. Deontic logics were strongly influenced by notions from modal logic. Despite the analogy between modal and deontic concepts can be traced to the XIV century (cf. McNamara 2006), it can be argued that the symbolic and mathematical approach to deontic logics was inaugurated in the celebrated paper (von Wright 1951). In that article, von Wright distinguishes three kinds of modalities: alethic, epistemic and deontic. The first ones deal with concepts such as "necessary" and "possible"; the second ones concern notions as "verifiable" and "falsifiable", whereas the latter are related to notions such as "obligatory" and "permitted".

There exist several systems formalizing deontic logic (see for instance Prakken \& Sergot 1994). The basic system is called SDL - Standard Deontic Logic. The idea behind this logic is to extend the basic modal system, in which the usual box operator $\square$ for necessitation is substituted by $\mathrm{O}$ (meaning "it is obligatory that") by adding the restriction that conflicting obligations are not allowed. This restriction is formulated by means of axiom (O-E) below. The following axiomatization of SDL presented in Coniglio 2007 is appropriate to our purposes.

Definition 2.1. The logic SDL is defined over the language $O, \neg, \wedge, \vee, \rightarrow$ by means of the following:

Axiom schemas:

(Ax1)-(Ax10) from mbC (recall Definition 1.2) plus

(exp) $(\alpha \rightarrow(\neg \alpha \rightarrow \beta))$

$(\mathrm{O}-\mathrm{K}) \mathrm{O}(\alpha \rightarrow \beta) \rightarrow(\mathrm{O} \alpha \rightarrow \mathrm{O} \beta)$

(O-E) $O f_{\alpha} \rightarrow \mathbf{f}_{\alpha} \quad$ where $\mathbf{f}_{\alpha}={ }_{\text {def }}(\alpha \wedge \neg \alpha)$

Inference rules:

Principia 13(3): 299-326 (2009). 
(MP) $\frac{\alpha \quad \alpha \rightarrow \beta}{\beta}$

(O-Nec) $\frac{\vdash \alpha}{1 \mathrm{O} \alpha}$

From now on, the set of sentences generated by the language $O, \neg, \wedge, \vee, \rightarrow$ will be denoted by For ${ }^{\mathrm{O}}$.

The usual axiomatizations of SDL depart from the set of axioms formed by the instances in the deontic language of the classical tautologies (arguably, this is a lazy and inelegant way to present an axiomatic extension of classical logic) and then consider, instead of (O-E), the axiom schema

(D) $\mathrm{O} \alpha \rightarrow \neg \mathrm{O} \neg \alpha$

(usually called the Principle of Deontic Consistency) and the inference rules (MP) and (O-Nec). It is useful and convenient to consider the derived deontic operator $\mathrm{P} \alpha={ }_{\text {def }} \neg \mathrm{O} \neg \alpha$ (meaning " $\alpha$ is permitted" or " $\alpha$ is permissible"). Thus, (D) can be interpreted as follows: whatever is obligatory is permitted. Since classically $\neg \beta$ is equivalent to $\beta \rightarrow \mathbf{f}_{\alpha}$ (for any $\alpha$ and $\beta$ ) then (D) can be reformulated as:

(D*) $\neg(\mathrm{O} \alpha \wedge \mathrm{O} \neg \alpha)$

stating that conflicting obligations are always false. Moreover, it can be proved that (O-E), (D) and (D*) are equivalent in the presence of classical logic plus (O-K), (O-Nec) and (MP).

An interesting alternative axiomatization of SDL is proposed in Chellas 1980 by substituting (O-K) and (O-Nec) by the following:

(ROM) $\frac{\alpha \rightarrow \beta}{\mathrm{O} \alpha \rightarrow \mathrm{O} \beta}$

(OC) $(\mathrm{O} \alpha \wedge \mathrm{O} \beta) \rightarrow \mathrm{O}(\alpha \wedge \beta)$

(ON) OT

(OD) $\neg \mathrm{O} \perp$

where $T$ can be taken as a classical tautology, for instance $\alpha \vee \neg \alpha$. Indeed, (OD) is an alternative formulation of $\left(D^{*}\right)$, while $(\mathrm{ON})$ and $(\mathrm{O}-\mathrm{Nec})$ are closely related. By its turn, (O-K) can be derived from (ROM) and (OC).

Concerning semantics, and being deontic logics particular cases of modal logics, the well-known Kripke semantics of possible-worlds offer an appropriate framework.

Principia 13(3): 299-326 (2009). 
Definition 2.2. A (generalized) Kripke struture is a triple $\left\langle W, R,\left\{v_{w}\right\}_{w \in W}\right\rangle$ where:

1. $W$ is a non-empty set (of possible-worlds);

2. $R \subseteq W \times W$ is a relation (of accessibility) between worlds;

3. $\left\{v_{w}\right\}_{w \in W}$ is a family of mappings $v_{w}: F_{0 r} \mathrm{O} \rightarrow 2$ such that:

$$
\begin{aligned}
& \text { (k1) } v_{w}(\alpha \wedge \beta)=1 \text { iff } v_{w}(\alpha)=v_{w}(\beta)=1, \\
& \text { (k2) } v_{w}(\alpha \vee \beta)=0 \text { iff } v_{w}(\alpha)=v_{w}(\beta)=0, \\
& \text { (k3) } v_{w}(\alpha \rightarrow \beta)=0 \text { iff } v_{w}(\alpha)=1 \text { and } v_{w}(\beta)=0, \\
& \text { (k4) } v_{w}(\neg \alpha)=1 \text { iff } v_{w}(\alpha)=0, \\
& \text { (k5) } v_{w}(\mathrm{O} \alpha)=1 \text { iff } v_{w^{\prime}}(\alpha)=1 \text { for every } w^{\prime} \text { in } W \text { such that } w R w^{\prime} .
\end{aligned}
$$

Let $M=\left\langle W, R,\left\{v_{w}\right\}_{w \in W}\right\rangle$ be a a Kripke structure, $w \in W$ and $\alpha$ a sentence. We say that $\alpha$ is satisfied by $M$ in $w$, denoted by $M, w \Vdash \alpha$, if $v_{w}(\alpha)=1 ; \alpha$ is satisfiable in $M$ if $M, w \Vdash \alpha$ for some $w \in W$, and $\alpha$ is true in $M$, denoted by $M \Vdash \alpha$, if $M, w \Vdash \alpha$ for every $w \in W$. Given a class $\mathscr{K}$ of Kripke structures, a sentence $\alpha$ is valid (w.r.t. $\mathscr{K}$ ) if, for every $M \in \mathscr{K}, M \Vdash \alpha$; and $\alpha$ is satisfiable (w.r.t. $\mathscr{K}$ ) if it is satisfiable for some $M \in \mathscr{K}$. Finally, the consequence relation $\vDash_{\mathscr{K}}$ is defined as follows: $\Gamma \vDash_{\mathscr{K}} \alpha$ iff there exists a finite set $\left\{\alpha_{1}, \ldots, \alpha_{n}\right\} \subseteq \Gamma$ such that $\left(\alpha_{1} \wedge \ldots \wedge \alpha_{n}\right) \rightarrow \alpha$ is valid (w.r.t. $\mathscr{K})$.

Theorem 2.3. Let $\mathscr{K}_{\text {SDL }}$ be the class of Kripke strutures such that the accessibility relation $R$ is serial, that is: for every $w \in W$ there exists $w^{\prime} \in W$ such that $w R w^{\prime}$. Then SDL is sound and complete for $\mathscr{K}_{\text {SDL }}$, that is: $\Gamma \vdash_{\text {SDL }} \alpha$ iff $\Gamma \vDash_{\mathscr{K}_{\text {SDL }}} \alpha$.

Now it is convenient to establish a parallel between some metalogical notions from classical and deontic logics. Recall that in classical logic a contradictory theory is trivial, and vice-versa. On the other hand, as observed in da Costa \& Carnielli 1986, if a theory $\Gamma$ derives conflicting obligations $\mathrm{O} \alpha$ and $\mathrm{O} \neg \alpha$ in SDL then $\Gamma$ is deontically trivial, that is, $\Gamma \vdash \mathrm{O} \beta$ for every $\beta$ (and, a posteriori, it is trivial: $\Gamma \vdash \beta$ for every $\beta$ ). The converse also holds, and so deontic contradictoriness is equivalent to deontic triviality, transposing to the deontic context a situation from classical logic.

In the previous section it was shown that LFI's incorporate metalogical notions such as consistency into the object language. This suggest us that some axioms as (ON) and (OD) can be treated as metalogical notions which can be internalized in the object language. Adapting to the modal context the definitions presented in Section 1 , the following definitions are proposed: 


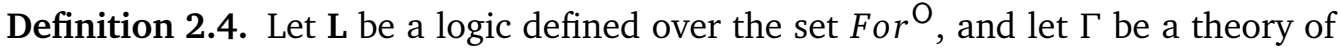
L. Then, $\Gamma$ is said to be deontically contradictory or deontically conflicting (w.r.t. O) if $\Gamma \vdash \mathrm{O} \alpha$ and $\Gamma \vdash \mathrm{O} \neg \alpha$ for some sentence $\alpha$. The theory $\Gamma$ is deontically trivial if $\Gamma \vdash$ $\mathrm{O} \alpha$ for every $\alpha$. If $\Gamma, \mathrm{O} \alpha, \mathrm{O} \neg \alpha \vdash \mathrm{O} \beta$ for every $\alpha$ and $\beta$ then $\Gamma$ is said to be deontically explosive. The logic $\mathbf{L}$ is deontically non-trivial if it has a deontically non-trivial theory, and it is deontically explosive if every theory is deontically explosive (equivalently, if $\mathrm{O} \alpha, \mathrm{O} \neg \alpha \vdash \mathrm{O} \beta$ for every $\alpha$ and $\beta$ ). The logic $\mathbf{L}$ is deontically paraconsistent if it is deontically non-trivial and deontically non-explosive.

It is worth noting that a deontic contradiction is of the form $\mathrm{O} \alpha \wedge \mathrm{O} \neg \alpha$ instead of $\mathrm{O} \alpha \wedge \neg \mathrm{O} \alpha$. Continuing with the analogy with LFI's, let $\bar{\boxminus}(p)$ be a set of sentences in $F_{0 r}{ }^{O}$ depending exclusively on the propositional letter $p$ such that, for some sentences $\alpha, \alpha^{\prime}, \beta$ and $\beta^{\prime}$,

(Da) $\bar{\boxminus}(\alpha), \mathrm{O} \alpha \nVdash \mathrm{O} \beta$

(Db) $\bar{\boxminus}\left(\alpha^{\prime}\right), \mathrm{O} \neg \alpha^{\prime} \nVdash \mathrm{O} \beta^{\prime}$

A logic $\mathbf{L}$ is deontically gently explosive (w.r.t. $\bar{\boxminus}(\alpha)$ ) if

(Dc) $\bar{\boxminus}(\alpha), \mathrm{O} \alpha, \mathrm{O} \neg \alpha \vdash \mathrm{O} \beta)$

for every $\alpha$ and $\beta$. Given a sentence $\alpha$, the set $\bar{\Xi}(\alpha)$ expresses the deontic consistence of $\alpha$ relative to logic $\mathbf{L}$ (and negation $\neg$ and obligation O). Whenever the set $\bar{\boxminus}(p)$ is a singleton, their unique element will be denoted by $\boxminus p$ and then the sentence $\boxminus \alpha$ denotes the deontic consistency of $\alpha$, where $\boxminus$ is called a deontic consistency operator. The following definition is the deontic version of Definition 1.1

Definition 2.5. A Logic of Deontic Inconsistency (LDI) w.r.t. $\mathrm{O}$ and $\neg$ is a deontically paraconsistent logic (w.r.t. $\mathrm{O}$ and $\neg$ ) having a set $\bar{\Xi}(p)$ satisfying properties (Da), $(\mathrm{Db})$ and $(\mathrm{Dc})$ as above.

It should be noticed that a deontically paraconsistent logic (w.r.t. a negation $\neg$ ) is necessarily paraconsistent (w.r.t. the same negation $\neg$ ). Indeed, by (exp), (O$\mathrm{Nec}$ ) and (O-K), an explosive deontic logic is, a posteriori, deontically explosive. Therefore, a deontically paraconsistent logic is, by force, a paraconsistent deontic logic.

On the other hand, it is possible to define paraconsistent deontic logics, that is, to enrich a given paraconsistent logic with a deontic operator, while preserving the property that conflicting obligations trivialize. Thus, a paraconsistent deontic logic is not necessarily a deontically paraconsistent logic. The analysis of systems of both types is the topic of the next section.

Principia 13(3): 299-326 (2009). 


\subsection{Paraconsistent Deontic Logics and Deontically Paraconsistent Logics}

The first paraconsistent deontic system proposed in the literature, called $C_{1}^{D}$, was introduced in da Costa \& Carnielli 1986 and it was also studied in Puga, da Costa \& Carnielli 1988. As suggested by its name, it is a deontic extension of $C_{1}$, in which some interesting relations between the consistency operator.$^{\circ}$ and the deontic operator $\mathrm{O}$ arise, as we shall see below.

Related to the approach mentioned above, in Cruz 2005 it was studied a deontic extension of $C_{1}$ but, instead of taking (O-E), a stronger version was considered: $\mathrm{O} \alpha \rightarrow \alpha$ (observe that (O-E) is a particular case, with $\alpha$ substituted by $\mathbf{f}_{\alpha}$ ). Another difference with the previous works is that the deontic operator $\mathrm{O}$ cannot be iterated, and so sentences such as $\mathrm{OO} \alpha$ and $\mathrm{O}(\alpha \rightarrow \mathrm{O} \beta)$ are not allowed. A paraconsistent dyadic modal logic was also considered, extending $C_{1}$ with a dyadic deontic operator $\mathrm{O}_{\beta} \alpha$ meaning " $\alpha$ is obligatory in context (or under condition) $\beta$ ". But, again, an excessively strong axiom was considered: $\mathrm{O}_{\beta} \alpha \rightarrow(\alpha \wedge \beta)$. Being so, some deontic paradoxes as Chisholm's paradox (see Section 3 below) cannot be satisfactorily treated in this framework.

In the rest of this section some paraconsistent deontic system will be analyzed.

\subsubsection{The logic DmbC}

In Coniglio 2007 a deontic dimension was added to mbC, a minimal LFI (recall Definition 1.2), by appropriately adapting the axiomatization of SDL presented in Definition 2.1. The resulting system, called DmbC, has the consistency operator $\circ$ as primitive (since it is based on $\mathbf{m b C}$ ) but no interaction between $\circ$ and $\mathrm{O}$ is required.

Definition 2.6. The logic DmbC is defined over the language $O, \circ, \neg, \wedge, \vee, \rightarrow$ by adding to $\mathrm{mbC}$ (recall Definition 1.2) the following:

Axiom schemas:

$(\mathrm{O}-\mathrm{K}) \mathrm{O}(\alpha \rightarrow \beta) \rightarrow(\mathrm{O} \alpha \rightarrow \mathrm{O} \beta)$

$(\mathrm{O}-\mathrm{E})^{\circ} \mathrm{O} \perp_{\alpha} \rightarrow \perp_{\alpha}$ where $\perp_{\alpha}={ }_{\text {def }}(\alpha \wedge \neg \alpha) \wedge \circ \alpha$

Inference rule:

(O-Nec) $\frac{\vdash \alpha}{\vdash \mathrm{O} \alpha}$

Alternatively, DmbC can be defined from the rules and axioms of SDL over For ${ }^{\circ} \mathrm{O}$ (the set of sentences generated by the language $O, \circ, \neg, \wedge, \vee, \rightarrow$ ) by replacing (exp)

Principia 13(3): 299-326 (2009). 
by (bc1) and (O-E) by (O-E) ${ }^{\circ}$, respectively. It can be proved that DmbC is both a LFI and a LDI. Thus, it has explicit operators for both consistency and deontic consistency. This fact will be proved in Theorem 2.8 below. The first step is to define a Kripke semantics for DmbC. This is obtained by modifying appropriately Definition 2.2 as follows:

Definition 2.7. A Kripke struture for $D m b C$ is a triple $\left\langle W, R,\left\{v_{w}\right\}_{w \in W}\right\rangle$ where:

1. $W$ is a non-empty set (of possible-worlds);

2. $R \subseteq W \times W$ is a relation (of accessibility) between worlds;

3. $\left\{v_{w}\right\}_{w \in W}$ is a family of mappings $v_{w}: F^{\circ} r^{\circ O} \rightarrow \mathbf{2}$ such that:

$$
\begin{aligned}
& \text { (kc1) } v_{w}(\alpha \wedge \beta)=1 \text { iff } v_{w}(\alpha)=v_{w}(\beta)=1, \\
& \text { (kc2) } v_{w}(\alpha \vee \beta)=0 \text { iff } v_{w}(\alpha)=v_{w}(\beta)=0, \\
& \text { (kc3) } v_{w}(\alpha \rightarrow \beta)=0 \text { iff } v_{w}(\alpha)=1 \text { and } v_{w}(\beta)=0, \\
& \text { (kc4) } v_{w}(\neg \alpha)=0 \text { implies } v_{w}(\alpha)=1, \\
& \text { (kc5) } v_{w}(\alpha)=v_{w}(\neg \alpha)=1 \text { implies } v_{w}(\circ \alpha)=0, \\
& \text { (kc6) } v_{w}(\mathrm{O} \alpha)=1 \text { iff } v_{w^{\prime}}(\alpha)=1 \text { for every } w^{\prime} \text { in } W \text { such that } w R w^{\prime} .
\end{aligned}
$$

Soundness and completeness of DmbC w.r.t. the semantics above was proved in Coniglio 2007. As a matter of fact, this result, as well as a similar result for system SDmbC to be defined below, are particular cases of a general completeness theorem stated in Bueno-Soler 2008 for logics based on LFI's enjoying the so-called $G^{k, l, m, n}$ axiom (cf. Carnielli \& Pizzi 2008).

Theorem 2.8. DmbC is both a LFI and a LDI.

Proof. Consider a Kripke model with $W=\{w\}, w R w$ and $v_{w}$ such that $v_{w}(p)=$ $v_{w}(\neg p)=1$ and $v_{w}(q)=0$ for propositional letters $p \neq q$. Then it is easy to see that $p, \neg p \nVdash_{\mathrm{DmbC}} q$ and $\mathrm{O} p, \mathrm{O} \neg p \nVdash_{\mathrm{DmbC}} \mathrm{O} q$, and so DmbC is neither explosive nor deontically explosive. In particular, DmbC is neither trivial nor deontically trivial. Using axiom (bc1) it follows that DmbC is a LFI in which the consistency of $\alpha$ is expressed by $\circ \alpha$. Finally, let $\boxminus \alpha={ }_{d e f}$ O $\alpha$. By (O-Nec) applied to (bc1), followed by (O-K) and (MP) it follows that DmbC is a LDI in which the deontic consistency of $\alpha$ is expressed by $\boxminus \alpha$.

It is easy to see that by adding to DmbC the axiom schema $\circ \alpha$ it is obtained a version of SDL over For ${ }^{\circ}$, denoted by eSDL. In fact, as proved above for mbC, 
axiom (exp) follows in that axiomatic extension of DmbC. On the other hand, by (O-Nec), $\mathrm{O} \circ \alpha$ is a theorem for every $\alpha$ and so (O-E) follows.

As observed in Carnielli, Coniglio \& Marcos 2007, the inconsistency operator • can be defined in $\mathrm{mbC}$ as $\bullet \alpha={ }_{\text {def }} \sim \circ \alpha$ in terms of the classical negation $\sim$, where $\sim \alpha=_{\text {def }}\left(\alpha \rightarrow \perp\right.$ ) (the expected definition $\bullet \alpha={ }_{\text {def }} \neg \circ \alpha$ in terms of the paraconsistent negation $\neg$ just works in stronger systems). Inspired by this, it is possible to define in DmbC an operator for deontic inconsistency as follows:

$$
\bigotimes \alpha={ }_{\text {def }} \mathrm{O} \sim \circ \alpha
$$

Since $\circ \alpha, \sim \circ \alpha \vdash \perp$ then $\mathrm{O} \circ \alpha, \mathrm{O} \sim \circ \alpha \vdash \mathrm{O} \perp$ and so $\boxminus \alpha, \nabla \alpha \vdash \perp$, by (O-E) ${ }^{\circ}$. This means that $\unrhd \alpha \vdash \sim \boxminus \alpha$ and $\boxminus \alpha \vdash \sim \otimes \alpha$, but the converses are not true (as it can be easily proved by using Kripke structures). Thus, the analogy between $\bullet$ and $\otimes$ is not complete: $\bullet \alpha \equiv \sim \circ \alpha$ and $\circ \alpha \equiv \sim \bullet \alpha$, but neither $\otimes \alpha \equiv \sim \boxminus \alpha$ nor $\boxminus \alpha \equiv \sim \otimes \alpha$.

\subsubsection{The logic DPI}

Observe that deontic consistency is a derived connective in DmbC (as in some other systems to be defined below). A natural question is: it would be possible to define the deontic counterpart of $\mathbf{m b C}$, in the sense of being a LDI such that deontic paraconsistency is expressed by a primitive connective? This logic would be non-explosive but without having a consistency connective. In other words, we are looking for a minimal LDI which is not a LFI.

At this point, we must recall that $\mathrm{mbC}$, the minimal LFI, has just one axiom for the consistency operator $\circ$, namely (bc1). By removing that axiom (and by removing $\circ$ from the language) it is recovered the interesting paraconsistent logic PI introduced in Batens 1980.

Definition 2.9. The logic PI is defined over the basic language $\neg, \wedge, \vee, \rightarrow$ as follows: Axiom schemas: Axioms (Ax1)-(Ax10) (recall Definition 1.2)

Inference rule: (MP) (recall Definition 1.2)

The logic PI can be transformed in a LDI by adding the modality $\mathrm{O}$, a connective for deontic consistency and appropriate rules and axioms, as follows.

Definition 2.10. The logic DPI is defined over the language $O, \boxminus, \neg, \wedge, \vee, \rightarrow$ by adding to PI the following:

Axiom schemas:

$(\mathrm{O}-\mathrm{K}) \mathrm{O}(\alpha \rightarrow \beta) \rightarrow(\mathrm{O} \alpha \rightarrow \mathrm{O} \beta)$ 
(Dbc1) $\boxminus \alpha \rightarrow(\mathrm{O} \alpha \rightarrow(\mathrm{O} \neg \alpha \rightarrow \mathrm{O} \beta))$

Inference rules:

(MP) $\frac{\alpha \quad \alpha \rightarrow \beta}{\beta}$

(O-Nec) $\frac{\vdash \alpha}{\vdash \mathrm{O} \alpha}$

Concerning semantics, DPI can be characterized by Kripke structures as those of DmbC, but now $R$ is not necessarily serial, and the valuations must satisfy clauses (kc1)-(kc4) and (kc6) of Definition 2.7, plus the following:

(kc5.1) $v_{w}(\boxminus \alpha)=v_{w}(\mathrm{O} \alpha)=v_{w}(\mathrm{O} \neg \alpha)=1$ implies $v_{w}(\mathrm{O} \beta)=1$ for every $\beta$.

Using the usual techniques, it can be proved that DPI is sound and complete w.r.t. its Kripke semantics.

As observed in Carnielli \& Marcos 2002, the logic PI is not a LFI and the same applies to DPI. On the other hand, by using Kripke structures it is easy to see that DPI is deontically paraconsistent, being obviously a LDI. So, the following result follows:

Theorem 2.11. DPI is a LDI but it is not a LFI.

The semantics for DPI deserves some comments. Firstly, it should be observed that it is possible to have a trivial valuation $v_{\text {true }}$ for PI such that $v_{\text {true }}(\alpha)=1$ for every $\alpha \in$ For. This is the semantical counterpart of the fact that PI is not finitely trivializable, and so there is no bottom formula, that is, a sentence $\perp$ such that $\perp \vdash_{\text {PI }} \alpha$ for every $\alpha$. Being so, it would be expected that its deontic extension DPI should maintain this feature and so it would be possible to satisfy $\mathrm{O} \alpha$ for every $\alpha$. This is why the accessibility relation of its Kripke frames is not necessarily serial: it is possible to have an isolated world $w$ in which $v_{w}(\alpha)=1$ (and, in particular, $\left.v_{w}(\mathrm{O} \alpha)=1\right)$ for every $\alpha \in$ For $^{\mathrm{O}}$.

The lack of a bottom formula (and so the absence of a consistency operator) prevents the definition of a strong (that is, a explosive) negation $\sim$ in both PI and DPI. Thus, the logic DPI is a relatively weak logic system.

\subsubsection{The logic SDPI}

From the last analysis, it seems that the logic DPI could be strengthened. A stronger deontic extension of PI is possible, closer to SDL, by allowing logical explosion from conflicting, deontically consistent obligations; in particular, this would allow the definition of a bottom formula $\perp$. It is enough to slightly modify DPI as follows:

Principia 13(3): 299-326 (2009). 
Definition 2.12. The logic SDPI is defined over the language $O, \boxminus, \neg, \wedge, \vee, \rightarrow$ by substituting in DPI axiom (Dbc1) by the following:

(SDbc1) $\boxminus \alpha \rightarrow(\mathrm{O} \alpha \rightarrow(\mathrm{O} \neg \alpha \rightarrow \beta))$

The Kripke semantics for SDPI consists of the Kripke structures of DPI satisfying the following clause:

(kc5.2) $v_{w}(\mathrm{O} \alpha)=v_{w}(\mathrm{O} \neg \alpha)=1$ implies $v_{w}(\boxminus \alpha)=0$.

Note that $(\mathrm{kc5} .1)$ is a consequence of $(\mathrm{kc5.2})$. It is straightforward to prove that SDPI is sound and complete w.r.t. its Kripke semantics.

It should also be noted that SDPI admits the definition of a bottom formula: indeed, $\perp_{\alpha}={ }_{\text {def }}(\mathrm{O} \alpha \wedge \mathrm{O} \neg \alpha) \wedge \boxminus \alpha$ is such that $\perp_{\alpha} \vdash_{\text {SDPI }} \beta$ for every $\alpha$ and $\beta$. However, it is still possible to define a Kripke structure for SDPI with an isolated world $w$ such that $v_{w}(\mathrm{O} \alpha)=1$ for every $\alpha$ (and, in particular, with $v_{w}\left(\mathrm{O} \perp_{\alpha}\right)=1$ ). This is why the accessibility relation is not required to be serial for SDPI, despite having a bottom formula definable in it.

By using any bottom $\perp$, it is possible to define a strong (that is, explosive) negation in SDPI as $\sim \alpha={ }_{\text {def }}(\alpha \rightarrow \perp)$. Note that

$$
\mathrm{O} \sim \alpha \vdash_{\text {SDPI }} \mathrm{O} \neg \alpha \text { but } \mathrm{O} \neg \alpha \nvdash_{\text {SDPI }} \mathrm{O} \sim \alpha \text {. }
$$

Moreover (and this is a general fact of paraconsistent logics having a bottom formula), SDPI is a LFI such that the consistency operator is defined $a$ la da Costa as follows: $\circ \alpha={ }_{\text {def }}(\alpha \wedge \neg \alpha) \rightarrow \perp$. That is, $\circ \alpha=\sim(\alpha \wedge \neg \alpha)$.

Theorem 2.13. SDPI is both a LFI and a LDI. Moreover, conflicting obligations involving a deontically consistent sentence trivialize.

This shows that this system has a reasonable expressive power, despite being based on a LFI weaker than mbC. Arguably, SDPI could be considered as the minimal paraconsistent version of SDL or, more precisely, of eSDL*, the version of SDL over For ${ }^{\mathrm{O}}$ obtained from SDPI by adding the axiom schema $\boxminus \alpha$. As expected, the accessibility relation of any Kripke structure for eSDL $^{*}$ must be serial.

To finalize, it can be observed that while DmbC and SDPI are both LFI's and LDI's, they are different in nature. While the former has the consistency operator $\circ$ as a primitive and the deontic consistency operator is derived, the latter follows the opposite way: the deontic operator $\boxminus$ is primitive, and the consistency operator is derived. 


\subsubsection{The logic $C_{1}^{D}$}

Now we shall briefly analyze $C_{1}^{D}$. As mentioned above, $C_{1}^{D}$ is obtained from $C_{1}$ by adding some deontic axioms. Recall that $C_{1}$ is defined over the basic language For (where $\alpha^{\circ}={ }_{\text {def }} \neg(\alpha \wedge \neg \alpha)$ ) by means of rule (MP), axioms (Ax1)-(Ax10) (see Definition 1.2) and:

(bC1) $\alpha^{\circ} \rightarrow(\alpha \rightarrow(\neg \alpha \rightarrow \beta))$

(ca1) $\left(\alpha^{\circ} \wedge \beta^{\circ}\right) \rightarrow(\alpha \wedge \beta)^{\circ}$

(ca2) $\left(\alpha^{\circ} \wedge \beta^{\circ}\right) \rightarrow(\alpha \vee \beta)^{\circ}$

(ca3) $\left(\alpha^{\circ} \wedge \beta^{\circ}\right) \rightarrow(\alpha \rightarrow \beta)^{\circ}$

The last three axioms state that consistency is "propagated" through the connectives (it can be proved that $\left.\alpha^{\circ} \rightarrow(\neg \alpha)^{\circ}\right)$. Different to mbC, the classical negation is defined as $\sim \alpha=_{\text {def }} \neg \alpha \wedge \alpha^{\circ}$.

Definition 2.14. The logic $C_{1}^{D}$ is defined over language $F_{o r}{ }^{\mathrm{O}}$ by adding to $C_{1}$ the following:

\section{Axiom schemas:}

$(\mathrm{O}-\mathrm{K}) \mathrm{O}(\alpha \rightarrow \beta) \rightarrow(\mathrm{O} \alpha \rightarrow \mathrm{O} \beta)$

(O-D) $\mathrm{O} \alpha \rightarrow \sim \mathrm{O} \sim \alpha$

(ca4) $\alpha^{\circ} \rightarrow(\mathrm{O} \alpha)^{\circ}$

Inference rule:

(O-Nec) $\frac{\vdash \alpha}{\vdash \mathrm{O} \alpha}$

Despite being formally analogous to axiom (D) of SDL, axiom (O-D) is equivalent to $(\mathrm{O}-\mathrm{E})^{\circ}$ of $\mathrm{DmbC}$, at it can be easily proved. Thus, the conception of $C_{1}^{D}$ is close to that of DmbC. It could be said that the modal extension of $C_{1}$ producing $C_{1}^{D}$ is in essence the same modal extension of $\mathbf{m b C}$ producing $\mathrm{DmbC}$ : the only additional modal axiom is (ca4), concerning the preservation of consistency through the operator $\mathrm{O}$. But this makes sense, because the preservation of consistency through the connectives is a general principle of $C_{1}$. By defining deontic consistency as $\boxminus \alpha={ }_{\text {def }} \mathrm{O}\left(\alpha^{\circ}\right)$, it can be proved the following:

Theorem 2.15. $C_{1}$ is both a LFI and a LDI.

Principia 13(3): 299-326 (2009). 


\subsubsection{The logic SDmbC}

The next step is the presentation of deontic systems which are LFI's but are not LDI's. This is the case of SDmbC and BDmbC, introduced in (Peron \& Coniglio 2008). As we shall see, the former is a paraconsistent logic deontically explosive; the latter is a bimodal deontic system, being deontically explosive with respect to one modality, and deontically paraconsistent with respect to the other.

Definition 2.16. The logic SDmbC is defined over For ${ }^{\circ O}$ by replacing in DmbC (recall Definition 2.6) axiom $(\mathrm{O}-\mathrm{E})^{\circ}$ by

$(\mathrm{O}-\mathrm{E})^{*}$ Of $f_{\alpha} \rightarrow \perp_{\alpha}$ where $\mathbf{f}_{\alpha}=(\alpha \wedge \neg \alpha)$ and $\perp_{\alpha}=(\alpha \wedge \neg \alpha) \wedge \circ \alpha$

It is worth noting that SDmbC is a paraconsistent deontic logic, but it is not a deontically paraconsistent logic (cf. Theorem 2.17 below). As in the case of DmbC, if the axiom schema $\circ \alpha$ is added to SDmbC then it is obtained eSDL, the version of SDL over $F_{0}{ }^{\circ}$. This situation is analogous to the relation between $\mathbf{m b C}$ and $\mathbf{~ C L L}$, the version of $\mathbf{C L}$ over For ${ }^{\circ}$, described in Section 1.

With respects to the semantics for SDmbC, it is enough to extend the Kripke semantics for DmbC (cf. Definition 2.7) by adding to the valuation mappings the following clause:

(kc7) $v_{w}(\mathrm{O} \neg \alpha)=1$ implies $v_{w^{\prime}}(\alpha)=0$ for every $w^{\prime}$ in $W$ such that $w R w^{\prime}$.

Clause (kc7) expresses that it is impossible to have conflicting obligations in a world $w$. As shown in (Peron 2009), the following results hold:

\section{Theorem 2.17.}

(i) SDmbC is a LFI.

(ii) SDmbC is deontically explosive and so it is not a LDI.

Theorem 2.18. SDmbC is sound and complete with respect to its Kripke semantics.

Another interesting feature of SDmbC is that the strong negation $\sim$ and the paraconsistent negation $\neg$ collapse within the scope of the deontic operator.

Theorem 2.19. $\mathrm{O} \neg \alpha \dashv$ SDmbc $\mathrm{O} \sim \alpha$. 


\subsubsection{The logic BDmbC}

The systems DmbC and SDmbC can be interesting in the analysis of deontic paradoxes, as we shall see in the next section. However, a logic combining both systems can enrich this analysis. Such a logic, called BDmbC, is obtained by combining DmbC and SDmbC, in a way that one deontic operator behaves as in DmbC, whereas the other behaves as in SDmbC.

The idea of considering paraconsistent bimodal deontic logics is not new. In Puga, da Costa \& Carnielli 1988 a bimodal system defined over $C_{1}$ called $\mathbb{C}_{1}$ having a deontic operator $\mathrm{O}$ and an alethic operator $\square$ was presented. Some interactions between both operators where forced, namely $\mathrm{O} \alpha \rightarrow \diamond \alpha$ (where, as usual, $\diamond \alpha={ }_{\text {def }}$ $\neg \square \neg \alpha$ is the "possibility" operator) and $\square \alpha \rightarrow \mathrm{O} \alpha$. This principles are based on ideas by Kant and Hintikka, respectively. Since $\square \alpha \rightarrow \alpha$ is an axiom of $\mathbb{C}_{1}$ then this logic is both a LFI and a LDI with respect to both modalities, where consistency and alethic consistency of $\alpha$ are expressed by the same sentence, namely $\alpha^{\circ}$; on the other hand, the deontic consistency of $\alpha$ is expressed by $\mathrm{O}\left(\alpha^{\circ}\right)$.

Our proposal, however, is different, by several reasons. On the one hand, both modalities of BDmbC are deontic, in contrast to $\mathbb{C}_{1}$ in which one modality is alethic. On the other hand, BDmbC is deontically explosive with respect to one modality and deontically paraconsistent with respect to the other, in contrast to $\mathbb{C}_{1}$ in which both modalities are not explosive, as mentioned above.

Definition 2.20. The logic BDmbC - Bimodal Deontic $m b C$ - is defined in the set For ${ }^{\circ \mathrm{OO}}$ of sentences generated by $\overline{\mathrm{O}}, \mathrm{O}, \circ, \neg, \wedge, \vee, \rightarrow$ by adding to $\mathrm{mbC}$ the following:

\section{Axiom schemas:}

$(\mathrm{O}-\mathrm{K}) \mathrm{O}(\alpha \rightarrow \beta) \rightarrow(\mathrm{O} \alpha \rightarrow \mathrm{O} \beta)$

$(\overline{\mathrm{O}}-\mathrm{K}) \overline{\mathrm{O}}(\alpha \rightarrow \beta) \rightarrow(\overline{\mathrm{O}} \alpha \rightarrow \overline{\mathrm{O}} \beta)$

$(\mathrm{O}-\mathrm{E})^{\circ} \mathrm{O} \perp_{\alpha} \rightarrow \perp_{\alpha}$ where $\perp_{\alpha}={ }_{\text {def }}(\alpha \wedge \neg \alpha) \wedge \circ \alpha$

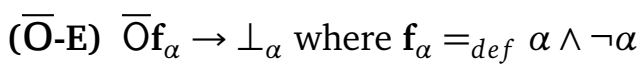

(BA) $\overline{\mathrm{O}} \alpha \rightarrow \mathrm{O} \alpha$

Inference rules:

(O-NEC) $\frac{\vdash \alpha}{\vdash \mathrm{O} \alpha}$

(D-NEC) $\frac{\vdash \alpha}{\vdash \overline{\mathrm{O}} \alpha}$

Principia 13(3): 299-326 (2009). 
The operator $\overline{\mathrm{O}}$ can be interpreted as "classically obligatory"; this notion is expressed in ( $\overline{\mathrm{O}}-\mathrm{E})$. On the other hand, $\mathrm{O}$ is interpreted as "weakly obligatory", as expressed in (O-E) ${ }^{\circ}$. However, an additional interaction between both operators is intended: namely, it is convenient that $\bar{O}$ be "stronger" or "stricter" than $O$, which justifies the inclusion of (BA). This axiom was inspired by the analogous one presented in the bimodal system KT ${ }^{\square}$, cf. Carnielli \& Pizzi 2008.

The logic BDmbC suggest an additional analogy between LFI's and LDI's. Just as $\mathrm{mbC}$ is $\neg$-paraconsistent but it is not $\sim$-paraconsistent, the theorem below shows that BDmbC is O-paraconsistent but it is not $\overline{\mathrm{O}}$-paraconsistent. The details of the proof are left to the reader.

Theorem 2.21.

(i) BDmbC is a LFI.

(ii) BDmbC is a LDI with respect to $\mathrm{O}$.

(iii) $\mathrm{BDmbC}$ is deontically explosive with respect to $\overline{\mathrm{O}}$ and so it is not a LDI with respect to $\overline{\mathrm{O}}$.

As expected, the semantics for BDmbC is given by Kripke structures having two accessibility relations, one for each modality. In details:

Definition 2.22. A Kripke structure for $B D m b C$ is a tuple $\left\langle W, R, \bar{R},\left\{v_{w}\right\}_{w \in W}\right\rangle$ where:

1. $W$ is a non-empty set (of possible-worlds);

2. $R \subseteq W \times W$ and $\bar{R} \subseteq W \times W$ are relations (of accessibility) between worlds which are serial;

3. $R \subseteq \bar{R}$;

4. $\left\{v_{w}\right\}_{w \in W}$ is a family of mappings $v_{w}:$ For $^{\circ \mathrm{OO}} \rightarrow 2$ satisfying clauses (kc1kc6) of Definition 2.7 plus the following clauses:

(kc6.1) $v_{w}(\overline{\mathrm{O}} \alpha)=1$ iff $v_{w^{\prime}}(\alpha)=1$ for every $w^{\prime}$ in $W$ such that $w \bar{R} w^{\prime}$,

(kc7.1) $v_{w}(\overline{\mathrm{O}} \neg \alpha)=1$ implies $v_{w^{\prime}}(\alpha)=0$ for every $w^{\prime}$ in $W$ such that $w \bar{R} w^{\prime}$.

The proof of the completeness of BDmbC with respect to its Kripke semantics can be found in Peron 2009. Since in BDmbC the operator $\mathrm{O} \alpha$ behaves as in DmbC, as long as $\overline{\mathrm{O}}$ has the same behavior as the deontic operator of SDmbC, it is easy to prove (by semantical means) the following result:

Principia 13(3): 299-326 (2009). 
Theorem 2.23. In BDmbC:

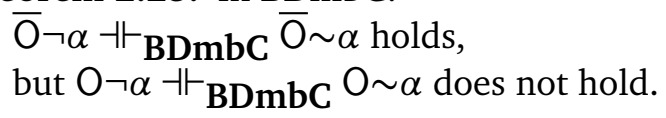

Of course it is possible to consider a plethora of paraconsistent deontic logics and LDI's by taking different LFI's and/or with combinations of (O-E) and (O-E) ${ }^{\circ}$ (as it was done with BDmbC). In particular, in (Coniglio 2007) the system DLFI1 based on the logic LFI1 was considered. The latter is a LFI enjoying nice features, for instance some axioms propagating the inconsistency operator $\bullet$. Another interesting point about LFI1 is that it has a simple 3-valued matrix semantics. The deontic system DLFI1 is both a LFI and a LDI such that the deontic inconsistency operator $\otimes$ satisfies the following: $\otimes \alpha \leftrightarrow(\mathrm{O} \alpha \wedge \mathrm{O} \neg \alpha)$. Moreover, $\otimes$ propagates under certain circumstances, which is an useful tool in the analysis of deontic paradoxes. The property of propagation of inconsistency resembles the propagation of consistency enjoyed by $C_{1}^{D}$. However, the underlying paraconsistent logics $C_{1}$ and LFI1 are quite different: while the latter is a 3-valued logic, the former cannot be characterized by finite matrices. Moreover, the propagation properties are not the same.

A previous and related approach to the analysis of modal paradoxes by using modal LFI's can be found in Costa-Leite 2003, where a paraconsistent alethic system called $\mathbf{C i}^{T}$ was proposed in order to analyze Fitch's paradox of knowability. The system $\mathbf{C i}^{T}$ is is a modal extension of $\mathbf{C i}$, a LFI introduced in Carnielli \& Marcos 2002.

Instead of defining more and more paraconsistent deontic systems and LDI's, the next section will analyze a classical deontic paradox, the so-called Chisholm's paradox, in the context of some of the system considered in this section.

\section{Chisholm's Paradox}

In general, a deontic paradox consists of a set of sentences in natural language, intuitively consistent and without logical dependencies such that, when formalized in a deontic language, it is logically trivial or it has logical dependencies. This situation occurs more frequently when the given sentences refer to norms, laws and moral principles: in this case contradictions can arise. Thus, most of such paradoxes are originated when the given set of premises is formalized in SDL as a set $\Gamma$ of logically independent sentences, but conflicting obligations such as $\mathrm{O} \alpha$ and $\mathrm{O} \neg \alpha$ are derived from $\Gamma$ within SDL. As pointed out in (McConnell 2006), the problem is related to the difficulties of SDL for dealing with contrary-to-duty obligations.

In this section, the well-known Chisholm's deontic paradox will be analyzed under the light of some LDI's discussed in the previous section. The aim is to show to what extent the paraconsistentist approach to deontic paradoxes can be fruitful. 
One of the first deontic paradoxes was proposed in Chisholm 1963. The following formulation of it was given in Åqvist 2002.

Consider the set $\mathscr{C}$ formed by the following sentences:

(1) It ought to be that John does not impregnate Suzy Mae.

(2) Not-impregnating Suzy Mae commits John to not marrying her.

(3) Impregnating Suzy Mae commits John to marry her.

(4) John impregnates Suzy Mae.

Let A and B be propositional letters representing the sentences "John impregnates Suzy Mae" and "John marries Suzy Mae", respectively. It is possible to formulate the set (1)-(4) above in a deontic language in several ways. In order to simplify the presentation, the following notions will be useful.

Definition 3.1. Consider in Dmbc the following derived deontic operators:

(i) $\mathrm{F}_{1} \alpha={ }_{\text {def }} \mathrm{O} \neg \alpha$ (prima-facie prohibition)

(ii) $\mathrm{F}_{2}={ }_{\text {def }} \mathrm{O} \sim \alpha$ (strong prohibition)

The name of such operators is inspired by Ross 1930 .

Returning to the set $\mathscr{C}$, it is clear that (1) admits two formulations in Dmbc: $F_{1} A$ and $F_{2} A$. By its turn, (2) has three interpretations: $\neg A \rightarrow F_{1} B, \neg A \rightarrow F_{2} B$ and $\mathrm{O}(\neg \mathrm{A} \rightarrow \neg \mathrm{B})$. Analogously, sentence (3) can be formalized as $\mathrm{A} \rightarrow \mathrm{OB}$ or $\mathrm{O}(\mathrm{A} \rightarrow \mathrm{B})$. Being so, there exist 12 possibilities to formalize the set $\mathscr{C}$, which are conveniently arranged below:

$$
\begin{aligned}
& \Gamma_{1.1}=\left\{F_{1} A, \neg A \rightarrow F_{1} B, A \rightarrow O B, A\right\} \\
& \Gamma_{1.2}=\left\{F_{1} A, \neg A \rightarrow F_{2} B, A \rightarrow O B, A\right\} \\
& \Gamma_{1.3}=\left\{F_{2} A, \neg A \rightarrow F_{1} B, A \rightarrow O B, A\right\} \\
& \Gamma_{1.4}=\left\{F_{2} A, \neg A \rightarrow F_{2} B, A \rightarrow O B, A\right\} \\
& \Gamma_{2.1}=\left\{F_{1} A, O(\neg A \rightarrow \neg B), A \rightarrow O B, A\right\} \\
& \Gamma_{2.2}=\left\{F_{2} A, O(\neg A \rightarrow \neg B), A \rightarrow O B, A\right\} \\
& \Gamma_{3.1}=\left\{F_{1} A, O(\neg A \rightarrow \neg B), O(A \rightarrow B), A\right\} \\
& \Gamma_{3.2}=\left\{F_{2} A, O(\neg A \rightarrow \neg B), O(A \rightarrow B), A\right\} \\
& \Gamma_{4.1}=\left\{F_{1} A, \neg A \rightarrow F_{1} B, O(A \rightarrow B), A\right\}
\end{aligned}
$$




$$
\begin{aligned}
& \Gamma_{4.2}=\left\{F_{1} A, \neg A \rightarrow F_{2} B, O(A \rightarrow B), A\right\} \\
& \Gamma_{4.3}=\left\{F_{2} A, \neg A \rightarrow F_{1} B, O(A \rightarrow B), A\right\} \\
& \Gamma_{4.4}=\left\{F_{2} A, \neg A \rightarrow F_{2} B, O(A \rightarrow B), A\right\}
\end{aligned}
$$

Clearly some of the sets above are not appropriate because some logical dependencies are present. This is exactly the paradoxical aspect of the set $\mathscr{C}$, because it is consistent and the sentences are logically independent. Now we will analyze Chisholm's paradox in some of the deontic systems defined above.

\subsection{Analysis in SDL}

Given that in SDL just one 'prohibition' operator is definable in SDL (because just classical negation is present) then the unique candidates to formalize $\mathscr{C}$ in SDL are $\Gamma_{1.4}, \Gamma_{2.2}, \Gamma_{3.2}$ and $\Gamma_{4.4}$. But the following laws hold in SDL:

$$
\vdash_{\mathrm{SDL}} \alpha \rightarrow(\neg \alpha \rightarrow \mathrm{O} \beta) \text { and } \vdash_{\mathrm{SDL}} \mathrm{O} \neg \alpha \rightarrow \mathrm{O}(\alpha \rightarrow \beta) .
$$

Then, (2) and (4) are logically dependent in $\Gamma_{1.4} ;(1)$ and (3) are logically dependent in $\Gamma_{3.2}$; by its turn, (1) and (3), on the one hand, and (2) and (4), on the other, are logically dependent in $\Gamma_{4.4}$.

So, the unique alternative in SDL is $\Gamma_{2.2}$. But then OB follows by (MP) between (3) and (4). On the other hand, $\vdash_{\text {SDL }} \mathrm{O}(\neg \mathrm{A} \rightarrow \neg \mathrm{B}) \rightarrow(\mathrm{O} \neg \mathrm{A} \rightarrow \mathrm{O} \neg \mathrm{B})$ and so, by (MP) two times it follows $\mathrm{O} \neg \mathrm{B}$. That is, the set $\Gamma_{2.2}$ is logically trivial in SDL, arriving so to a paradox.

\subsection{Analysis in DmbC}

Moving now to DmbC, there are much more alternatives for choosing a set $\Gamma_{i . j}$, because there are less logical interdependencies.

By taking appropriate Kripke structures, it is easy to prove that

$$
\nvdash_{\mathrm{DmbC}} \alpha \rightarrow(\neg \alpha \rightarrow \mathrm{O} \neg \beta) \text { and } \quad \nvdash_{\mathrm{DmbC}} \alpha \rightarrow(\neg \alpha \rightarrow \mathrm{O} \sim \beta) \text {. }
$$

Moreover, by using Kripke structures it is easy to see that the sentences in $\Gamma_{1.1}, \Gamma_{1.2}$, $\Gamma_{1.3}$ and $\Gamma_{1.4}$ are logically independent in DmbC. The independence of the premises in $\Gamma_{2.1}, \Gamma_{2.2}, \Gamma_{3.1}, \Gamma_{4.1}$ and $\Gamma_{4.2}$ is obtained by analogous arguments. By (O-K) and (MP), OB is derived from $\Gamma_{1 . j}$ (for $1 \leq j \leq 4$ ) and also from $\Gamma_{2.1}$ and $\Gamma_{2.2}$. By a similar argument, $\mathrm{O} \neg \mathrm{B}$ is derived from $\Gamma_{i . j}$ (for $i . j=2.1,2.2,3.1$ ). This means that $\Gamma_{2.1}$ and $\Gamma_{2.2}$ are deontically contradictory. 
Concerning the set $\Gamma_{3.2}$, note that $\vdash_{\text {DmbC }} \sim A \rightarrow(A \rightarrow B)$ and then, by (O-Nec) and (O-K) there is a dependence between (1) and (3). Then, this set cannot be used for the analysis. By the same reason the sets $\Gamma_{4.3}$ and $\Gamma_{4.4}$ must be discarded.

Altogether, there are nine sets logically independent in DmbC formalizing $\mathscr{C}$ and so being potentially useful for the analysis of Chisholm's paradox, in contrast to SDL which have just one. Remarkably, none of them are logically trivial and so the nine sets are relevant for the analysis. It is easy to prove by using Kripke structures that $\Gamma_{i . j}$ does not deduce $\mathrm{O} \neg \mathrm{B}$ for $i . j=1.1,1.2,1.3,1.4,4.1,4.2$. By its turn, $\Gamma_{i . j}$ does not deduce OB for $i . j=3.1,4.1,4.2$. Thus, all these sets do not infer conflicting obligations. Finally, $\Gamma_{i . j}$ deduce conflicting obligations for $i . j=2.1,2.2$, but there is no deontic explosion (and so there is no logical explosion).

Summarizing, in DmbC we have the following scenarios for Chisholm's paradox:

$$
\begin{aligned}
& \Gamma_{i . j} \vdash_{\text {DmbC }} \text { OB but } \Gamma_{i . j} \nvdash_{\text {DmbC }} \mathrm{O} \neg \mathrm{B} \text { for } i . j=1.1,1.2,1.3,1.4 \\
& \Gamma_{3.1} \vdash_{\text {DmbC }} \mathrm{O} \neg \mathrm{B} \text { but } \Gamma_{3.1} \nvdash_{\text {DmbC }} \text { OB } \\
& \Gamma_{i . j} \vdash_{\text {DmbC }} \text { OB, O } \neg \mathrm{B} \text { without trivializing deontically, for } i . j=2.1,2.2 \\
& \Gamma_{i . j} \nvdash_{\text {DmbC }} \text { OB and } \Gamma_{i . j} \nvdash_{\text {DmbC }} \mathrm{O} \neg \mathrm{B} \text { for } i . j=4.1,4.2
\end{aligned}
$$

That is, DmbC has enough expressive power to encompass all the possible situations, namely:

- just $O B$ is derived;

- just $\mathrm{O} \neg \mathrm{B}$ is derived;

- both $\mathrm{OB}$ and $\mathrm{O} \neg \mathrm{B}$ are derived, without having deontic explosion (and so, without trivializing);

- neither $\mathrm{OB}$ nor $\mathrm{O} \neg \mathrm{B}$ are derived.

\subsection{Analysis in SDmbC}

As happens in SDL, in the logic SDmbC the deontic operators $F_{1}$ and $F_{2}$ collapse; let $F$ be the unique prohibition operator in SDmbC. Thus, there are just four possibilities in SDmbC formalizing $\mathscr{C}$, namely:

$$
\begin{aligned}
& \Gamma_{0.1}=\{\mathrm{FA}, \neg \mathrm{A} \rightarrow \mathrm{FB}, \mathrm{A} \rightarrow \mathrm{OB}, \mathrm{A}\} \\
& \Gamma_{0.2}=\{\mathrm{FA}, \mathrm{O}(\neg \mathrm{A} \rightarrow \neg \mathrm{B}), \mathrm{A} \rightarrow \mathrm{OB}, \mathrm{A}\} \\
& \Gamma_{0.3}=\{\mathrm{FA}, \mathrm{O}(\neg \mathrm{A} \rightarrow \neg \mathrm{B}), \mathrm{O}(\mathrm{A} \rightarrow \mathrm{B}), \mathrm{A}\}
\end{aligned}
$$




$$
\Gamma_{0.4}=\{\mathrm{FA}, \neg \mathrm{A} \rightarrow \mathrm{FB}, \mathrm{O}(\mathrm{A} \rightarrow \mathrm{B}), \mathrm{A}\}
$$

It is easy to see that in SDmbC it holds:

$$
\nvdash_{\mathrm{SDmbC}} \alpha \rightarrow(\neg \alpha \rightarrow \mathrm{O} \beta) \text { but } \vDash_{\mathrm{SDmbC}} \mathrm{O} \neg \alpha \rightarrow \mathrm{O}(\alpha \rightarrow \beta)
$$

and so $\Gamma_{0.3}$ and $\Gamma_{0.4}$ are discarded because there are logical dependencies among the premises. On the other hand, $\Gamma_{0.2}$ trivializes in SDmbC, because this system does not accept conflicting obligations. Thus, the only admissible set is $\Gamma_{0.1}$ which satisfies the following:

$$
\Gamma_{0.1} \vdash_{\text {SDmbC }} \text { OB but } \Gamma_{0.1} \nvdash_{\text {SDmbC }} \mathrm{O} \neg \mathrm{B} \text {. }
$$

That is, Chisholm's paradox can again be avoided, producing the expected result: John has to marry Suzy Mae.

\subsection{Analysis in DPI and in SDPI}

Since DPI admits just one negation, namely the paraconsistent one, just the sets $\Gamma_{0 . j}$ (for $j=1, \ldots, 4$ ) of SDmbC are obtained, with $F \alpha=\mathrm{O} \neg \alpha$. As expected, $\Gamma_{0.1}$ just derives OB; $\Gamma_{0.3}$ just derives $\mathrm{O} \neg \mathrm{B} ; \Gamma_{0.2}$ derives both $\mathrm{OB}$ and $\mathrm{O} \neg \mathrm{B}$ without deontic explosion (and so without trivializing); and $\Gamma_{0.4}$ neither derives $\mathrm{OB}$ nor derives $\mathrm{O} \neg \mathrm{B}$.

Concerning SDPI, the same results obtained for DmbC hold in this logic, mutatis mutandis.

\subsection{Analysis in BDmbC}

The logic BDmbC validates all the inferences in DmbC about O, as well as the inferences of SDmbC about $\overline{\mathrm{O}}$. Moreover, three prohibition operators can be defined: $\mathrm{O} \neg \alpha, \mathrm{O} \sim \alpha$ and $\overline{\mathrm{O}} \neg \alpha$. Since two obligation operators are available, namely $\mathrm{O} \alpha$ and $\overline{\mathrm{O}} \alpha$, there are much more alternatives to formalize the set $\mathscr{C}$. Being an extension of DmbC, all the possible situations for the paradox described in Subsection 3.2 can also be obtained in BDmbC. However, since there are two kinds of obligations in BDmbC, there are strictly more possible situations than in DmbC. Namely, the following obligations concerning $B$ are obtained from the different sets of premises:

- just $O B$ is derived;

- just $\overline{\mathrm{OB}}$ (and so $\mathrm{OB}$ ) is derived;

- just $\mathrm{O} \neg \mathrm{B}$ is derived;

- just $\overline{\mathrm{O}} \neg \mathrm{B}$ (and so $\mathrm{O} \neg \mathrm{B}$ ) is derived; 
- $\mathrm{OB}$ and $\mathrm{O} \neg \mathrm{B}$ are derived, without having deontic explosion;

- $\overline{\mathrm{O}} \mathrm{B}$ (and so $\mathrm{OB}$ ) and $\mathrm{O} \neg \mathrm{B}$ are derived, without having deontic explosion;

- neither $\mathrm{OB}($ nor $\overline{\mathrm{O}} \mathrm{B})$ nor $\mathrm{O} \neg \mathrm{B}($ nor $\overline{\mathrm{O}} \neg \mathrm{B}$ ) are derived;

- everything is derived (logical explosion).

\subsection{Summarizing}

To summarize, all the plausible answers to the question "Should John marry Suzy Mae?" are obtained in DmbC, DPI and SDPI. In the case of BDmbC, there are still more variants for the answers (even the logical explosion can be attained), as shown above. On the other hand, SDmbC produces just one answer, the intuitively expected one. In contrast, SDL just produces logical explosion (or logical dependencies).

The main point of our analysis lies in the fact that Chisholm's paradox is not exclusively based on the violation of the Principle of Deontic Consistency as presented frequently in the literature (this position is defended, for instance, in (Prakken \& Sergot 1994)). The analysis above suggests that the logical dependence between the premises in $\mathscr{C}$ (dependency arising because of the use of classical logic) plays an important role. Thus, in SDmbC the principle of deontic explosion is maintained while rejecting the principle of explosion, and then it is possible to avoid the paradox. That is, by eliminating some logical dependencies typical of classical logic, the paradox vanishes. In the case of DmbC, DPI and SDPI, both principles of explosion are weakened and so there are more possibilities to avoid the paradox. Finally, BDmbC combines DmbC and SDmbC, obtaining still more solutions to the paradox by combination of its deontic operators.

\section{Final remarks}

Our analysis departs from the transposition of the approach to paraconsistency of LFI's to the deontic context. This allows to consider notions such as deontic explosion and deontic paraconsistency.

The Logics of Deontic (In)Consistency seem to encompass several paraconsistent deontic systems already proposed in the literature, suggesting the definition of a taxonomy of such systems, in a similar way as the proposal for LFI's started in Carnielli \& Marcos 2002.

The approach to Chisholm's paradox presented here is based on the generality of LDI's, and so it is possible to obtain solutions to the paradox based on the lack of logical dependencies and/or the elimination of the principle of deontic explosion. The distinction between the two sources of paradox can be useful to analyze other 
deontic paradoxes, as those presented in Prakken \& Sergot 1994, Prakken \& Sergot 1997 and Carmo \& Jones 1997.

Clearly, the research on paraconsistent deontic logic and deontic paradoxes has several possibilities of further development. We hope that our discussion can contribute to some extent to the analysis of the interesting question of deontic paradoxes and related areas. ${ }^{1}$

\section{References}

Åqvist, L. 2002. Deontic logic. In D. Gabbay and F. Guenthner (eds.) Handbook of Philosophical Logic (2nd. edition), volume 8, 147-264. Kluwer Academic Publishers.

Batens, D. 1980. Paraconsistent extensional propositional logics. Logique et Analyse 90-91: 195-234.

Bueno-Soler, J. 2008. Possible-translations semantics for cathodic modal logics. CLE e-Prints 8(6).

$\mathrm{URL}=$ http://www.cle.unicamp.br/e-prints/vol_8,n_6, 2008.html.

Carmo, J. \& Jones, A. 1997. Dyadic deontic logic and contrary-to-duty obligations. In D. Nute (ed.) Defeasible Deontic Logic, volume 263 of Synthese Library, 317-44. Kluwer Publishing Company.

Carnielli, W. A.; Coniglio, M.; Marcos, J. 2007. Logics of Formal Inconsistency. In D. Gabbay and F. Guenthner (eds.) Handbook of Philosophical Logic (2nd. edition), volume 14, 15107. Springer.

Carnielli, W. A. \& Marcos, J. 1999. Limits for paraconsistency calculi. Notre Dame Journal of Formal Logic 40(3): 375-90.

2002. A Taxonomy of C-systems. In: Carnielli, W. A., Coniglio, M. E. and D’Ottaviano, I. M. L. (eds.) Paraconsistency: The Logical Way to the Inconsistent, pp. 1-94, Marcel Dekker. Preliminary version available at CLE e-Prints 1(5), 2001.

$\mathrm{URL}=$ http://www.cle.unicamp.br/e-prints/abstract_5.htm.

Carnielli, W. A. \& Pizzi, C. 2008. Modalities and Multimodalities, volume 12 of Logic, Epistemology, and the Unity of Science. Springer Verlag.

Chellas, B. 1980. Modal Logic: an introduction. Cambridge: Cambridge University Press.

Chisholm, R. 1963. Contrary-to-duty imperatives and Deontic Logic. Analysis 24: 33-6.

Coniglio, M. E. 2007. Logics of Deontic Inconsistency. CLE e-Prints 7(4). $\mathrm{URL}=$ http://www.cle.unicamp.br/e-prints/vol_7,n_4,2007.html.

Costa-Leite, A. 2003. Paraconsistência, modalidade e cognoscibilidade (Paraconsistency, Modality and Cognoscibility, in Portuguese). Master's thesis, Institute of Philosophy and Human sciences, State University of Campinas (UNICAMP), Campinas.

Creswell, M. J. \& Hughes, G. E. 1996. A New Introduction to Modal Logic. London: Houtledge.

Cruz, A. M. P. 2005. Lógica Deôntica Paraconsistente: Paradoxos e Dilemas (Paraconsistent Deontic Logic: Paradoxes and Dilemmas, in Portuguese). Natal: Editora da UFRN, Brazil.

da Costa, N. C. A. 1963. Sistemas Formais Inconsistentes (Inconsistent Formal Systems, in Portuguese). Habilitation Thesis. Republished by Editora UFPR, Curitiba, 1993.

- 1974. On the theory of inconsistent formal systems. Notre Dame Journal of Formal Logic XV: 497-510.

Principia 13(3): 299-326 (2009). 
da Costa, N. C. A. \& Carnielli, W. A. 1986. Paraconsistent Deontic Logic. Philosophia 16(3/4): 293-305.

Jaśkowski, S. 1948. Rachunek zdan dla systemów dedukcyjnych sprzecznych. Societatis Scientiarun Torunesis, Sectio A I: 55-77. Translated as: "Propositional Calculus for Contradictory Deductive Systems", Studia Logica 24: 143-157, 1969.

McConnell, T. 2006. Moral Dilemmas. In E. N. Zalta (ed.), The Stanford Encyclopedia of Philosophy.

URL = http://www.plato.stanford.edu/entries/modal-dilemmas/.

McNamara, P. 2006. Deontic Logic. In E. N. Zalta (ed.) The Stanford Encyclopedia of Philosophy.

$\mathrm{URL}=$ http://www.plato.stanford.edu/entries/logic-deontic/.

Peron, N. M. 2009. Lógicas da Inconsistência Deôntica (Logics of Deontic Inconsistency, in Portuguese). Master's thesis, Institute of Philosophy and Human sciences, State University of Campinas (UNICAMP), Campinas.

Peron, N. M. \& Coniglio, M.E. 2008. Logics of Deontic Inconsistencies and Paradoxes. CLE e-Prints 8(6).

$\mathrm{URL}=$ http://www.cle.unicamp.br/e-prints/vol_8,n_6,2008.html.

Prakken, H. \& Sergot, M. 1994. Contrary-to-duty imperatives, defeasibility and violability. In: Jones, A. and Sergot, M. (eds.), Proceedings of the 2nd International Workshop on Deontic Logic in Computer Science (DEON94), volume 1/94 of CompLex, pp. 296-318, Tano A.S., Oslo.

- 1997. Dyadic deontic logic and contrary-to-duty obligations. In: Nute, D. (ed.), Defeasible Deontic Logic, volume 263 of Synthese Library, pp. 223-62. Kluwer Publishing Company.

Puga, L.Z., da Costa, N. C. A. \& Carnielli, W. A. 1988. Kantian and Non-Kantian Logics. Logique \& Analyse 31(121/122): 3-9.

Ross, D. 1930. The Right and the Good. Oxford: Oxford University Press.

von Wright, G. 1951. Deontic Logic. Mind 60: 1-15. Republished in: Logical Studies (by G. H. von Wright). Londres: Routledge and Kegan Paul, 1967, 58-74.

Marcelo Esteban Coniglio and Newton Marques Peron Department of Philosophy and Centre for Logic, Epistemology and the History of Science (CLE) State University of Campinas (UNICAMP)

P.O. Box 6110 13081-970 Campinas, SP, Brazil coniglio@cle.unicamp.br and newton.peron@gmail.com

Resumo. As Lógicas da (In)Consistência Deôntica (LDI's) podem ser consideradas como sendo a contraparte deôntica das lógicas paraconsistentes chamadas de Lógicas da (In)Consistência Formal. Neste artigo são introduzidas e estudadas novas LDI's e outras lógicas deônticas paraconsistentes satisfazendo diferentes propriedades: sistemas tolerantes a obrigações contraditórias; sistemas em que as obrigações contraditórias produzem trivialização; e uma lógica deôntica paraconsistente bimodal que combina as características de sistemas previamente introduzidos. Estas lógicas são utilizadas para analisar o conhecido paradoxo de

Principia 13(3): 299-326 (2009). 
Chisholm aproveitando-se do fato de que, além que as obrigações contraditórias não trivializam nas LDI's, varias das dependências lógicas da lógica clássica são bloqueadas no contexto das LDI's, permitindo assim dissolver o paradoxo.

Palavras-chave: Lógica paraconsistente, lógica deôntica, paradoxos deônticos, paradoxo de Chisholm, dilemas morais, lógicas da inconsistência formal.

\section{Notes}

${ }^{1}$ The first author was supported by an individual research grant from The National Council for Scientific and Technological Development (CNPq), process \#304090/2008-5.

Principia 13(3): 299-326 (2009). 